\title{
Spectral Distributions of Thomson Scattered Photons from High-intensity Pulsed Lasers
}

\author{
G. A. Krafft \\ Thomas Jefferson National Accelerator Facility, 12000 Jefferson Ave., Newport \\ News, Virginia, 23606, USA
}

General formulas for the far-field spectral distribution of photons Thomson scattered by a single electron have been obtained. Effects due to the pulsed nature of the laser beam are explicitly allowed, simultaneously with intensity high enough that harmonic generation is possible. For realistic pulsed photon beams the spectrum of backscattered radiation is considerably broadened because of changes in the longitudinal velocity of the electrons during the pulse. Such ponderomotive broadening is especially pronounced at higher harmonics, eventually leading to a continuous emission spectrum.

The subject of Thomson scattering received attention many years ago in discussions of mass shifts of electrons in intense lasers ${ }^{12} 3$, and in studies of fusion plasma diagnostics ${ }^{4}$. Recently, the subject has been revived because of attempts to produce X-rays, particularly pulses with short time durations, by Thomson scattering from electron beams of energy of 10-100 $\mathrm{MeV}^{5}$. Such work is stimulated by the hope that a small accelerator might produce X-rays of energies of $1-10 \mathrm{keV}$ in modest university-scale facilities, but 
with much less coherent flux than is possible at the new "fourth generation light sources" based on Self-Amplified Spontaneous Emission free electron lasers (SASE FELs). As a result of previous work, there is much known about the scattering of continuous wave (CW) laser light at high intensity, obtained by simplifying the text-book solution of the classical motion of an electron in a plane wave ${ }^{6}$ to the case of constant continuous illumination, and using it to calculate the Thomson scattered power.

Roughly simultaneously and independently of this effort, accelerator light source physicists developed ways of calculating the photon yield from the interactions of electron beams with insertion devices. For example, they have considered electromagnetic radiation emission from short magnets, or magnets with a "sharp" field edge in order to study the optical synchrotron radiation generated by heavy particles at high energy. When applied to emission by an electron, an example of a result of relevance is ${ }^{7}$

$$
\frac{d E}{d \nu d \Omega}=\frac{r_{e}^{2} c}{\pi} \gamma^{2} f^{2}\left(1+\gamma^{2} \theta^{2}\right)^{2} \mid \widetilde{B}\left(\nu\left(1+\gamma^{2} \theta^{2}\right) / 2 \gamma^{2}\right)^{2}
$$

where $d E$ is the energy emitted by a single electron into frequency interval $d v$ and solid angle $d \Omega, r_{e}$ is the classical electron radius, $\gamma, \beta_{z}$, and $c$ are the usual relativistic factors, $\widetilde{B}\left(v^{\prime}\right)$ is the time Fourier transform (in the lab frame) of the magnetic field that the electron traverses, and $f^{2}=f_{\sigma}^{2}+f_{\pi}^{2}$ where 


$$
\begin{aligned}
& f_{\sigma}=\frac{1}{\left(1+\gamma^{2} \theta^{2}\right)^{2}} \sin \phi \\
& f_{\pi}=\frac{1}{\left(1+\gamma^{2} \theta^{2}\right)^{2}}\left(\frac{1-\gamma^{2} \theta^{2}}{1+\gamma^{2} \theta^{2}}\right) \cos \phi
\end{aligned}
$$

are the radiation distribution angular patterns at high $\gamma$ and small observation angle $\theta$, for the standard $(\sigma, \pi)$ polarizations. In these expressions $\theta$ and $\phi$ are the usual spherical coordinates with $z$-axis aligned along the electron longitudinal motion and $x$-axis aligned with the bend direction. It will be shown elsewhere, that for magnetic fields such that the local value of the field strength parameter $K=e B(z) \lambda / 2 \pi m c^{2}$ is much less than one, these distributions may be more generally written as

$$
\begin{aligned}
\frac{d E_{\sigma}}{d \omega d \Omega} & =\frac{r_{e}^{2}}{8 \pi^{2} c} \frac{\left|\widetilde{B}\left(\omega\left(1-\beta_{z} \cos \theta\right) / c \beta_{z}\right)\right|^{2}}{\gamma^{2}\left(1-\beta_{z} \cos \theta\right)^{2}} \sin ^{2} \phi \\
\frac{d E_{\pi}}{d \omega d \Omega} & =\frac{r_{e}^{2}}{8 \pi^{2} c} \frac{\mid \widetilde{B}\left(\omega\left(1-\beta_{z} \cos \theta\right) / c \beta_{z}\right)^{2}}{\gamma^{2}\left(1-\beta_{z} \cos \theta\right)^{2}}\left(\frac{\cos \theta-\beta_{z}}{1-\beta_{z} \cos \theta}\right)^{2} \cos ^{2} \phi
\end{aligned}
$$

where $e$ is the fundamental charge, $\lambda$ is the scale-length for changes in the magnetic field, $m$ is the particle mass, $\omega=2 \pi v$, and now $\widetilde{B}(k)$ indicates the space Fourier transform of the magnetic field. Note that these expressions include that fact that the accelerated electron is acted on by a field of finite spatial extent; in the beam frame the magnetic field appears to be pulsed. 
In a Thomson backscattering arrangement, i. e., when an electron beam collides with a laser beam head-on, there are equivalent expressions for the scattered energy distribution that apply at low field strengths ${ }^{8}$, including the fact that the laser beam is pulsed:

$$
\begin{aligned}
& \frac{d E_{\sigma}}{d \omega d \Omega}=\frac{r_{e}^{2}}{8 \pi^{2} c} \frac{\mid \widetilde{E}\left(\omega\left(1-\beta_{z} \cos \theta\right) /\left.c\left(1+\beta_{z}\right)\right|^{2}\right.}{\gamma^{2}\left(1-\beta_{z} \cos \theta\right)^{2}} \sin ^{2} \phi \\
& \frac{d E_{\pi}}{d \omega d \Omega}=\frac{r_{e}^{2}}{8 \pi^{2} c} \frac{\mid \widetilde{E}\left(\omega\left(1-\beta_{z} \cos \theta\right) /\left.c\left(1+\beta_{z}\right)\right|^{2}\right.}{\gamma^{2}\left(1-\beta_{z} \cos \theta\right)^{2}}\left(\frac{\cos \theta-\beta_{z}}{1-\beta_{z} \cos \theta}\right)^{2} \cos ^{2} \phi
\end{aligned}
$$

It is the purpose of the present paper to point out that a generalization of these results to high field strengths can be achieved. Comparing the Thomson back-scattering case and the insertion device result one observes two main differences: (1) the electron is being accelerated by the transverse electric field of the laser pulse instead of a transverse magnetic field, and (2) the frequency of oscillation (in the lab frame) is shifted up by $\left(1+\beta_{z}\right)$ in the photon-electron scattering case, as opposed to $\beta_{z}$ for insertion devices, because the photons are moving across the electrons in the lab frame in the former case, whereas the magnetic field is fixed in the lab frame in the latter case.

As the magnetic field strength parameter increases in an insertion device three basic changes occur in the electron orbit ${ }^{9}$. First, the average longitudinal velocity decreases to $\beta^{*}=1-\left(1+K^{2} / 2\right) / 2 \gamma^{2}$. Because of the reduced longitudinal velocity, the frequency spectrum is shifted down in frequency by the $1+K^{2} / 2$ factor. Secondly, because the magnitude of the velocity must remain fixed in the electron orbit in a static magnetic field, a velocity modulation in the longitudinal direction must arise as a result 
of the transverse velocity. This modulation, in the $z$-direction, which occurs at twice the frequency of the transverse motion, has the effect of yielding an orbit that appears as a figure 8 in the rest frame of the electron. The acceleration in $z$ generates dipole emission. Thirdly, as $K$ approaches one the motion becomes relativistic and it is no longer valid to neglect retardation-driven phase delays in evaluating the emitted radiation. When the phase delays are included, one observes that harmonics of the fundamental emission frequency are generated. Qualitatively, higher harmonics are generated the higher that $K$ is.

One expects similar behavior from the emission generated by an intense laser pulse. In the future, the detailed calculation will be presented. Here the calculation method and results are summarized. There is an exact solution to the problem of the classical electron motion in a plane-wave electromagnetic field ${ }^{6}$. This is easiest accomplished by solving the Hamilton-Jacobi equation for the motion under the correct boundary conditions, assuming that a plane-wave description of the incident laser pulse, further assumed to be linearly polarized and described by a single transverse component of the vector potential $A_{x}(\xi \equiv z+c t)$, is possible. The exact solution for the electron motion, in the lab frame,

with boundary conditions $\mathrm{x}(t \rightarrow-\infty)=\left(0,0, \beta_{z} c t\right)$ and $\mathrm{f}(t \rightarrow-\infty)=\left(0,0, \beta_{z} c\right)$ is

$$
\begin{gathered}
c t=\frac{\xi}{1+\beta_{z}}+\frac{1}{\gamma^{2}\left(1+\beta_{z}\right)^{2}} \int_{-\infty}^{\xi} \frac{e^{2} A_{x}^{2}\left(\xi^{\prime}\right)}{2 m^{2} c^{4}} d \xi^{\prime} \\
x=\frac{1}{\gamma\left(1+\beta_{z}\right)} \int_{-\infty}^{\xi} \frac{e A_{x}\left(\xi^{\prime}\right)}{m c^{2}} d \xi^{\prime}
\end{gathered}
$$




$$
\begin{gathered}
y=0 \\
z=\frac{\beta_{z} \xi}{1+\beta_{z}}-\frac{1}{\gamma^{2}\left(1+\beta_{z}\right)^{2}} \int_{-\infty}^{\xi} \frac{e^{2} A_{x}^{2}\left(\xi^{\prime}\right)}{2 m^{2} c^{4}} d \xi^{\prime}
\end{gathered}
$$

When a change of variables is made from time to the independent variable $\xi$ in the expression for the radiation produced by this electron motion ${ }^{10}$, an exact analytical expression for the scattered intensity may be obtained:

$$
\begin{gathered}
\frac{d E_{\sigma}}{d \omega d \Omega}=\frac{e^{2} \omega^{2} \mid D_{x}(\omega)^{2}}{8 \pi^{2} c^{3}} \sin ^{2} \phi \\
\frac{d E_{\pi}}{d \omega d \Omega}=\frac{e^{2} \omega^{2}}{8 \pi^{2} c^{3}}\left|D_{x}(\omega)\left(\frac{\cos \theta-\beta_{z}}{1-\beta_{z} \cos \theta}\right) \cos \phi+D_{z}(\omega) \sin \theta\right|^{2}
\end{gathered}
$$

where

$$
\begin{gathered}
D_{x}(\omega)=\int \frac{d \xi}{\gamma\left(1+\beta_{z}\right)} \frac{e A_{x}(\xi)}{m c^{2}} \exp i \omega\left(\begin{array}{c}
\frac{\xi\left(1-\beta_{z} \cos \theta\right)}{c\left(1+\beta_{z}\right)}-\frac{\sin \theta \cos \phi}{c \gamma\left(1+\beta_{z}\right)} \int_{-\infty}^{\xi} \frac{e A_{x}\left(\xi^{\prime}\right)}{m c^{2}} d \xi^{\prime} \\
+\frac{(1+\cos \theta)}{c \gamma^{2}\left(1+\beta_{z}\right)^{2}} \int_{-\infty}^{\xi} \frac{e^{2} A_{x}^{2}\left(\xi^{\prime}\right)}{2 m^{2} c^{4}} d \xi^{\prime}
\end{array}\right) \text { (2a) } \\
D_{z}(\omega)=\frac{1+\beta_{z}}{\left(1-\beta_{z} \cos \theta\right)} \int \frac{d \xi}{\gamma^{2}\left(1+\beta_{z}\right)^{2}} \frac{e^{2} A_{x}^{2}(\xi)}{2 m^{2} c^{4}} \exp i \omega\left(\begin{array}{c}
\frac{\xi\left(1-\beta_{z} \cos \theta\right)}{c\left(1+\beta_{z}\right)}-\frac{\sin \theta \cos \phi}{c \gamma\left(1+\beta_{z}\right)} \int_{-\infty}^{\xi} \frac{e A_{x}\left(\xi^{\prime}\right)}{m c^{2}} d \xi^{\prime} \\
+\frac{(1+\cos \theta)}{c \gamma^{2}\left(1+\beta_{z}\right)^{2}} \int_{-\infty}^{\xi} \frac{e^{2} A_{x}^{2}\left(\xi^{\prime}\right)}{2 m^{2} c^{4}} d \xi^{\prime}
\end{array}\right) .
\end{gathered}
$$


The only approximation in these expressions is the "far-field" approximation, i. e., that one observes at a distance large compared to the source size. Physically, the $D$ s give the effective frequency content generated by the electron velocity in both the $x$-direction and the $z$-direction, respectively, including the finite laser pulse effects that are implicit in a finitely extended $A_{x}(\xi)$, and including the fact that at large field amplitude the motion is both relativistic, and has phase shifts due to retardation. In the weak-field limit $D_{z} \ll D_{x}$, and $D_{x}$ is precisely the Fourier transform of the electron velocity in the $x$ direction, properly Doppler shifted as above. Given $A_{x}(\xi)$, it is straightforward to integrate these expressions to obtain the proper energy spectrum.

To make contact with standard results from undulators, it is worthwhile to consider the case $A_{x}(\xi)=A_{0} \cos \left(2 \pi \xi / \lambda_{0}\right)\left[\Theta(\xi)-\Theta\left(\xi-N \lambda_{0}\right)\right]$, where $\Theta(x)$ is the usual unit step function, yielding a hard-edge laser pulse with constant amplitude for $N$ wavelengths. Defining the field strength parameter $a \equiv e A_{0} / m c^{2}$, the lab-frame wave number $k_{0}=2 \pi / \lambda_{0}$, and following the prescription of Alferov, Bashmakov, and Bessonov ${ }^{11}$ for expressing the harmonic amplitudes in terms of Bessel Function sums, one obtains the following expressions for the energy content into each harmonic of the fundamental $n$,

$$
\frac{d E_{\sigma, n}}{d \omega d \Omega} \approx \frac{e^{2}}{2 c} \frac{\sin ^{2} \phi}{\sin ^{2} \theta \cos ^{2} \phi}\left[S_{1 n}+S_{2 n} / n\right]^{2} f_{n N}^{2}(\omega ; n \omega(\theta)),
$$


$\frac{d E_{\pi, n}}{d \omega d \Omega} \approx \frac{e^{2}}{2 c}\left[\begin{array}{c}S_{1 n}\left(\frac{\cos \theta-\beta_{z}}{\left(1-\beta_{z} \cos \theta\right) \sin \theta}+\frac{a^{2}}{4} \frac{\omega}{n \gamma\left(1+\beta_{z}\right) k_{0} c} \frac{\sin \theta}{\gamma\left(1-\beta_{z} \cos \theta\right)}\right) \\ +\frac{S_{2 n}}{n \sin \theta}\end{array}\right] f_{n N}^{2}(\omega ; n \omega(\theta))$,

where

$$
\begin{gathered}
S_{1 n} \equiv \sum_{k^{\prime}=-\infty}^{\infty} J_{n+2 k^{\prime}}\left(\frac{a \omega}{k_{0} c} \frac{\sin \theta \cos \phi}{\gamma\left(1+\beta_{z}\right)}\right) J_{k^{\prime}}\left(\frac{a^{2}}{8} \frac{\omega}{k_{0} c} \frac{(1+\cos \theta)}{\gamma^{2}\left(1+\beta_{z}\right)^{2}}\right), \\
S_{2 n} \equiv \sum_{k^{\prime}=-\infty}^{\infty} 2 k^{\prime} J_{n+2 k^{\prime}}\left(\frac{a \omega}{k_{0} c} \frac{\sin \theta \cos \phi}{\gamma\left(1+\beta_{z}\right)}\right) J_{k^{\prime}}\left(\frac{a^{2}}{8} \frac{\omega}{k_{0} c} \frac{(1+\cos \theta)}{\gamma^{2}\left(1+\beta_{z}\right)^{2}}\right), \\
\omega(\theta)=\frac{\left(1+\beta_{z}\right)\left(2 \pi c / \lambda_{0}\right)}{1-\beta_{z} \cos \theta+\left(a^{2} / 4\right)\left(1-\beta_{z}\right)(1+\cos \theta)},
\end{gathered}
$$

and

$$
f_{m}\left(\omega ; \omega^{\prime}\right) \equiv \sin \left(m \pi \omega / \omega^{\prime}\right) / \sin \left(\pi \omega / \omega^{\prime}\right)
$$

is highly peaked at $\omega=\omega^{\prime}$ with peak value $m$, and whose square integrates to $m \omega^{\prime}$ for large $m$ if the domain of the integral includes more than several bandwidths $\omega^{\prime} / m$ around $\pm \omega^{\prime}$. 
A numerical method, based on $10^{5}-10^{6}$ point Simpson integration of Eqn. (2a), has been used to investigate two cases where the laser pulse has many oscillations within a slowly varying envelope, $A_{x}(\xi)=\alpha_{x}(\xi) \cos \left(2 \pi \xi / \lambda_{0}\right)$. Motion spectra, $D_{x}(\omega)$, were calculated for constant $\alpha_{x}(\xi)=A_{0}, N=20, \gamma=100$, in the forward direction $\theta=0$, and for two constant values of the field strengths. The results are presented in Figure 1, with $a=0.02$ and $a=0.5$, respectively. The Figure gives $D_{x} / \lambda_{0}$ as a function of the scaled frequency $\omega / \omega_{0}$ where $\omega_{0}=\left(1+\beta_{z}\right)^{2} \gamma^{2} 2 \pi c / \lambda_{0}$ is the maximum frequency emitted, in the forward direction with $\theta \rightarrow 0$. One observes the standard result from undulator theory ${ }^{9}$, that emission is peaked at odd harmonics of the scaled frequency. By examining the first harmonic peaks in detail one observes the expected frequency shift to longer wavelengths by the factor $1+a^{2} / 2$ as the field strength is increased. Note however, that when the strength is constant in $z$, the relative width of the spectrum at each peak is the same at the two field strengths. In particular, the relative widths are narrower than the frequency shift entailed by going from $a=0.01$ to $a=0.5$. The accuracy of the numerical method was evaluated by comparing its results to the analytical results in Eqns. 3. The differences are at the $10^{-15}$ level in $D_{x}$; there is no significant difference between a million point Simpson integration and Eqns. 3.

Much more interesting is the result when a Gaussian laser pulse is considered. Figure 2 gives two spectra, calculated with $\alpha_{x}(\xi)=A_{\text {peak }} \exp \left(-\xi^{2} / 2\left(8.156 \lambda_{0}\right)^{2}\right)$, where $a_{\text {peak }}=0.0117$ and $a_{\text {peak }}=0.587$. The specific values were chosen so that the number of photons per pulse (per unit area) and the rms duration of the pulses are the same as in 
Figure 1. At low field strength the main peaks in the spectrum are very similar to those in Figure 1 above, with the satellite peaks from the interference from the sharp-edge distribution being eliminated with a Gaussian starting pulse. Even so, the high field strength case shows that the more realistic and smoother starting pulse yields very much weaker and broader spectra than before, a phenomenon that might be called "ponderomotive broadening". As the laser pulse travels by, the electron is first slowed, and then sped up by the ponderomotive effect from the laser pulse. These velocity shifts lead to frequency shifts in the emitted radiation, increasing the width of the observed spectrum. Because the electron spends a larger fraction of time in the "high-field" portion of the laser pulse, it will radiate most at the maximum red shift, explaining the qualitative observation that the maximum in the spectrum occurs at the maximum frequency shift at each harmonic. Also note that the relative frequency shift is the same for each harmonic. This means that at high enough harmonic number, the peaks must begin to overlap.

The broadening effect has direct relevance in a recent experiment ${ }^{12}$. In Figure 3 effective motion spectra are shown for three values of $a_{p e a k}$, for the laser conditions obtained from the reference. Because the scattering electrons were stationary in the lab frame in this experiment, $\gamma=1$, and the harmonic frequency scale is set by the incident laser frequency without a Doppler shift. In the calculation the longitudinal $\sigma$ for the intensity was approximately 10 laser oscillations (26 fsec for a Ti:Sa laser pulse). For $a_{\text {peak }}$ at the smallest value, individual harmonic lines are observed. For the middle value, just under 2 , the spectrum is continuous except for a dip just above the first harmonic line. For the highest $a_{\text {peak }}$ value, as in the experiment, the spectrum becomes continuous and in fact, 
high harmonics are generated with substantial strength. As is obvious from the frequency scaling of the results ${ }^{10}$, the frequencies of the harmonics are much greater than the corresponding synchrotron radiation critical frequency, even for the highest field strength case.

Ponderomotive broadening can clearly effectively limit the possible energy spectral density of Thomson emission to levels far below what one might expect based on simple scaling of constant-amplitude results. For example, suppose one had a 1000 period laser pulse. A priori, one might expect to be able to obtain sharp emission into a full spectral width of roughly $\Delta \omega / \omega \leq 1 / 1000=0.001$. Unfortunately, ponderomotive broadening can be ignored only if the field strength is low enough, that is if $a^{2} / 2<<\Delta \omega / \omega$, or if the pulse is very flat throughout the 1000 periods. At the other extreme, compressing optical pulses to obtain higher $a$ in a shorter pulse length may not substantially increase the energy spectral density of the emission because the ponderomotive detuning increases quickly with $a^{2}$.

In this paper we presented some results from a general theory for calculating the energy spectral distributions, at arbitrary observation angles, of the photons generated in Thomson backscattering events. The theory is more general than previous theories in that 
finite laser pulse length is allowed simultaneously with high effective field strength ${ }^{\mathrm{a}}$. The main input to the theory is the plane-wave vector potential describing the incident laser pulse because this function, and not the electric field, determines the motion including all relativistic effects. It should be noted that the vector potential function will be markedly different than the electric field function in situations where the laser amplitude changes greatly in a wavelength, a case covered in this general theory. The theory makes contact with, and can encompass, previously known results on spectra generated by high-field strength magnetic insertion devices. It allows many of the standard results from undulator theory to be carried over to analyzing Thomson backscattering photon source arrangements, and it shows that a previously unnoted phenomenon, ponderomotive broadening, can lead to reduced energy spectral density in some situations. The author acknowledges being stimulated to complete this work by the opportunity to present a course on Thomson Scattering X-ray Sources at the United States Particle Accelerator School. This work supported by the United States Department of Energy under Contract DE-AC05-84ER40150.

\section{References}

\footnotetext{
${ }^{\text {a }}$ For details see http://casa.jlab.org/publications/lecture_2003.shtml, especially the Jan. 14, 2003 lecture.
} 
${ }^{1}$ Lowell S. Brown and T. W. B. Kibble, Phys. Rev. 133, A704 (1964).

2 Joseph H. Eberly and Arthur Sleeper, Phys. Rev. 176, 1570 (1968).

3 E. S. Sarachik and G. T. Schappert, Phys. Rev. D 1, 2738 (1970).

${ }^{4}$ Cesar I. Castillo-Herrera and Tudor W. Johnston, IEEE Trans. Plasma Sci. 21, 125 (1993).

5 R. L. Schoenlein, W. P. Leemans, A. H. Chin, P. Volfbeyn, T. E. Glover, P. Balling, M. Zolotorev, K.-J. Kim, S. Chattopadhyay, and C. V. Shank, Science 274, 236 (1996).

${ }^{6}$ L. D. Landau and E. M. Lifshitz, The Classical Theory of Fields, 4th revised English edition (Pergamon, Oxford, 1975), pgs.112-114.

7 R. Coisson, Phys. Rev. A 20, 524 (1979).

${ }^{8}$ G. A. Krafft, Proceedings of the 1997 Particle Accelerator Conference, Vancouver, BC, 739, (1997). IEEE, Piscataway, NJ, (1998).

9 K.-J. Kim, American Institute of Physics Conference Proceedings (AIP, New York, 1989), Vol. 184, p.565.

10 J. D. Jackson, Classical Electrodynamics, 2nd edition (John Wiley, New York, (1975)), Chap. 14, p.671.

11 D. F. Alferov, Yu. A. Bashmakov, and E. G. Bessonov, Sov. Phys. Tech. Phys. 18, 1336 (1974).

${ }^{12}$ K. Ta Phuoc, A. Rousse, M. Pittman, J. P. Rousseau, V. Malka, S. Fritzler, D. Umstadter, and D. Hulin, Phys. Rev. Lett. 91, 195001 (2003). 


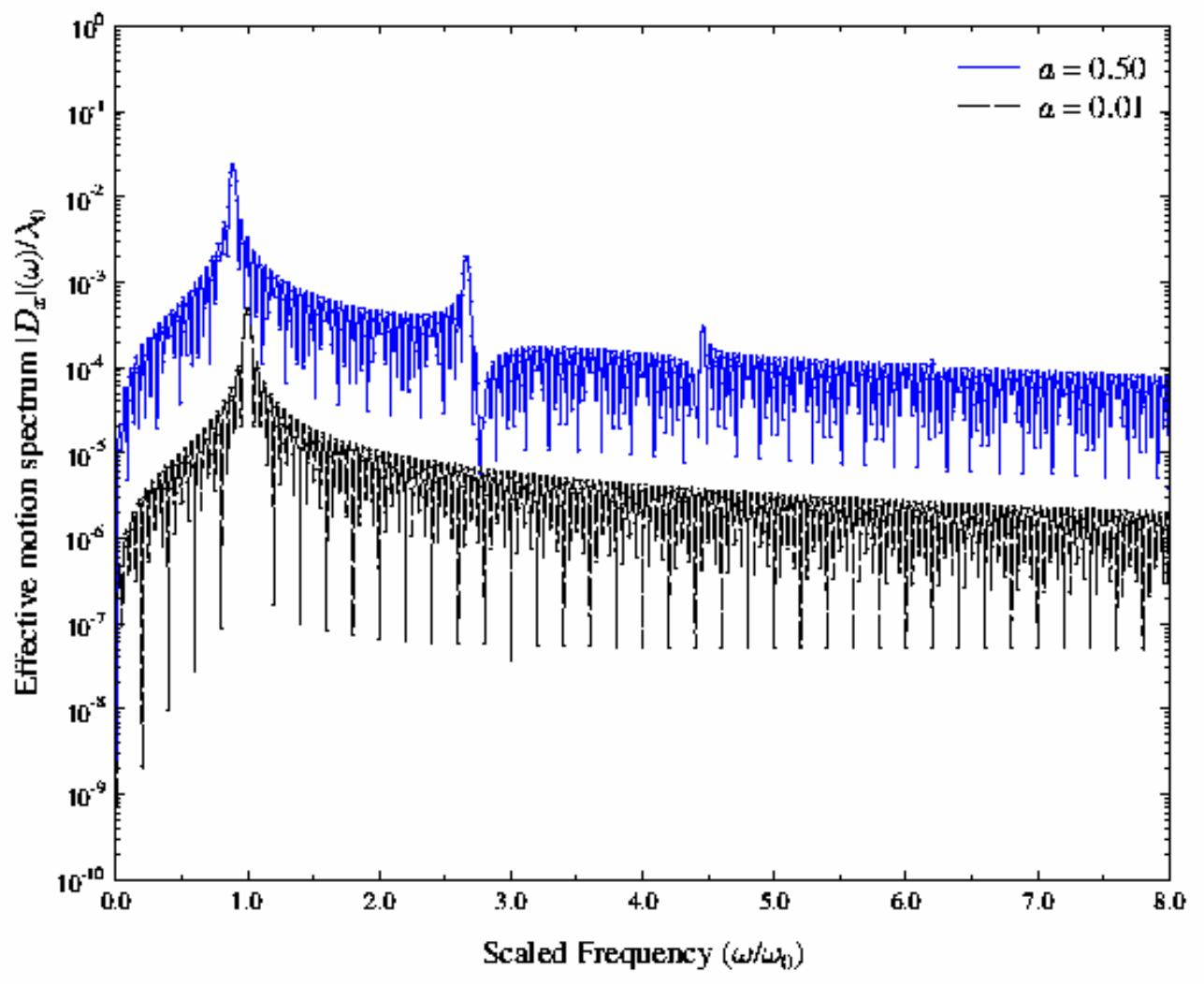

Figure 1 Effective motion spectra for 20 oscillation flat laser pulse at two values of the field strength for a $\gamma=\mathbf{1 0 0}$ electron beam. In order to reduce confusion in the $a=$ 0.50 plot, data at $\omega=0.4 i \omega_{0}$, for $i=1, \ldots, 19$, have been omitted from the plot. At these frequencies $D_{x}$ vanishes due to destructive interference. 


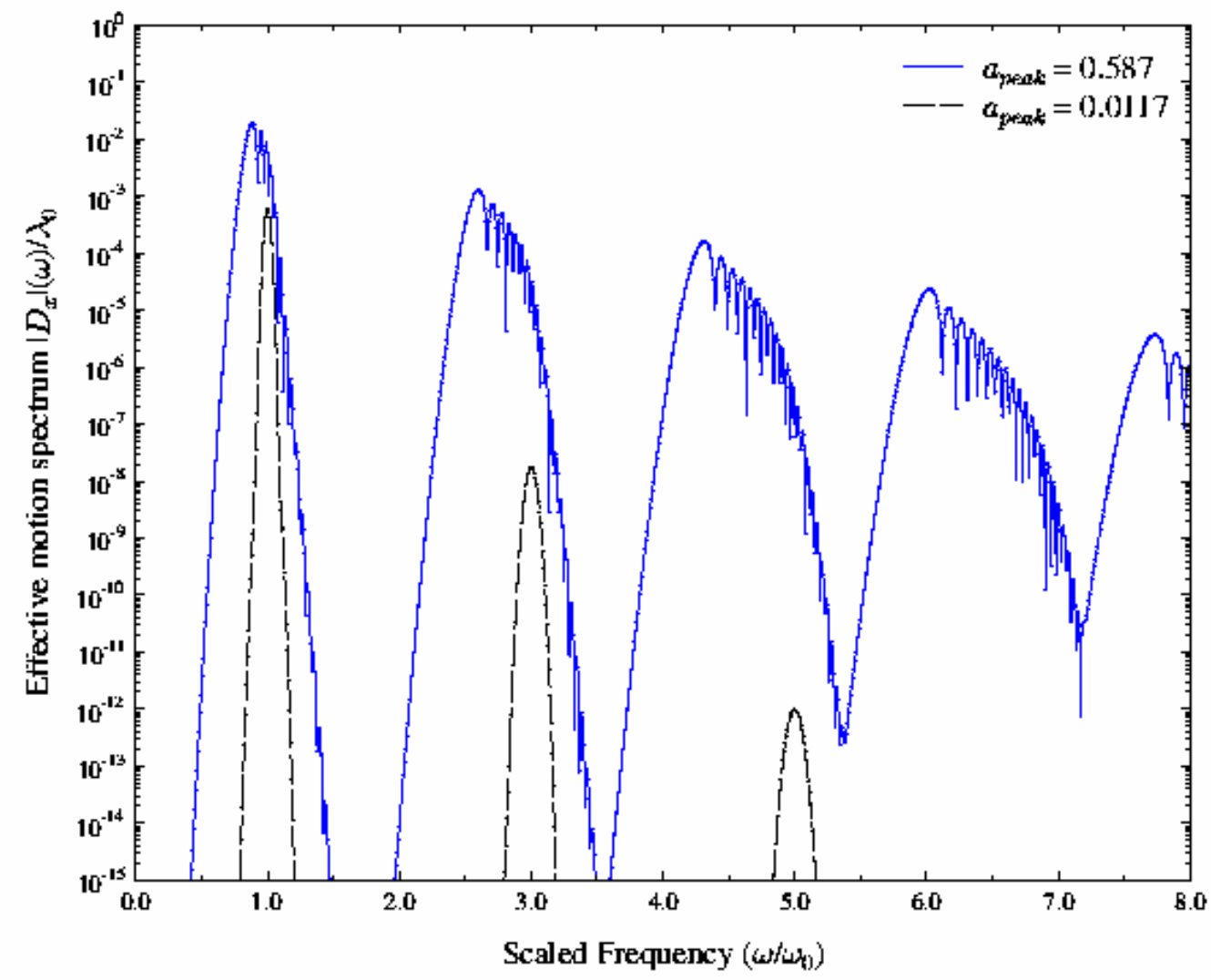

Figure 2 Effective motion spectra for a Gaussian laser pulse. The spectrum is broadened at high field strength due to ponderomotive effects, which change the electron longitudinal velocity. The dips within the spectral peaks are not numerical. They are due to destructive interference of the induced emission from various longitudinal locations within the laser pulse. 


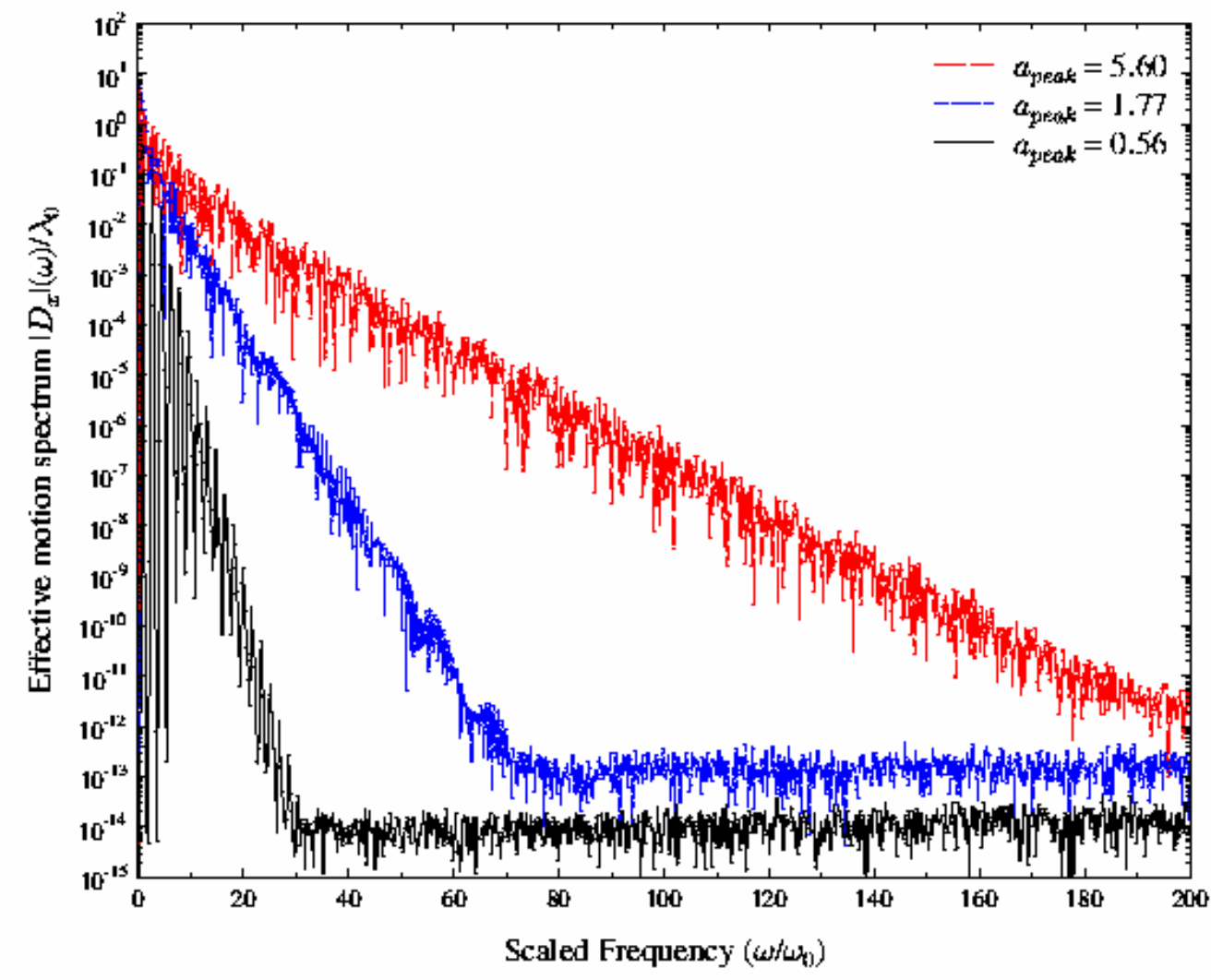

Figure 3 Thomson forward scatter spectrum from a recent experiment. The lowest field strength case has individual harmonic peaks as in Figure 2. The higher field spectra oscillate, are not harmonic, and scale as the synchrotron radiation spectrum above critical frequency. Flat regions are at the noise floor of the numerical calculations. 